\title{
Preoperative Staging in Women with Known Breast Cancer: Comparison between Digital Breast Tomosynthesis (DBT) and Magnetic Resonance Imaging (MRI)
}

\author{
Francesca Galati ${ }^{1}$, Flaminia Marzocca ${ }^{1}$, Andrea Tancredi ${ }^{2}$, Emmanuel Collalunga ${ }^{1}$, Carlo Catalano ${ }^{1} \&$ Federica \\ Pediconi ${ }^{1}$ \\ ${ }^{1}$ Department of Radiological, Oncological and Pathological Sciences, University of Rome «Sapienza», Rome, \\ Italy \\ ${ }^{2}$ Department of Methods and Models for Economics, Territory and Finance, University of Rome «Sapienza», \\ Rome, Italy \\ Correspondence: Francesca Galati, Department of Radiological, Oncological and Pathological Sciences, \\ "Sapienza" University of Rome, V.le Regina Elena, 324, 00161 Rome, Italy. Tel: 39-064-455-602. E-mail: \\ francesca.galati3@gmail.com
}

Received: September 26, 2018

Accepted: October 12, $2018 \quad$ Online Published: November 8, 2018

doi:10.5539/cco.v7n2p33

URL: https://doi.org/10.5539/cco.v7n2p33

\section{Abstract \\ Objectives}

To prospectively evaluate the accuracy in tumor extent and size assessment of Digital Breast Tomosynthesis (DBT) and Magnetic Resonance Imaging (MRI) in women with known breast cancer, with pathological size as the gold standard.

\section{Methods}

From May 2014 to April 2016, 50 patients with known breast cancer were enrolled in our prospective study. All patients underwent MRI on a 3T magnet and DBT projections. Two radiologists, with 15 and 7 years of experience in breast imaging respectively, evaluated in consensus each imaging set unaware of the final histological examination. MR and DBT sensitivity, PPV and accuracy were calculated, using histology as the gold standard. McNemar test was used to compare MR and DBT sensitivity. Correlation and regression analyses were used to evaluate MRI vs Histology, DBT vs Histology and MRI vs DBT lesions tumor size agreement to histological results.

\section{Results}

On histological examination 70 lesions were detected. MRI showed 100\% sensitivity, 96\% PPV and $96 \%$ accuracy; DBT sensitivity was $81 \%$, PPV $92 \%$ and accuracy $77 \%$. McNemar test p-value was 0.0003 . Lesions size Pearson correlation coefficient was 0.97 for MRI vs Histology, 0.92 for DBT vs Histology, (p-value<0.0001). MRI vs DBT regression coefficient was 0.83 .

\section{Conclusions}

MRI confirmed to be the most accurate imaging technique in preoperative staging of breast cancer. However, DBT showed very good accuracy, sensitivity and tumor size assessment and could be a valid tool for preoperative staging when MRI is contraindicated.

Keywords: digital breast tomosynthesis, magnetic resonance imaging, breast cancer, preoperative staging

\section{Abbreviations}

EUSOMA: European Society of Breast Cancer Specialists

FFDM: full field digital mammography

US: ultrasound

MRI: magnetic resonance imaging 
ILC: infiltrative lobular carcinoma

PBI: partial breast irradiation

DBT: digital breast tomosynthesis

NHSBSP: National Health Service Breast Screening Programme

\section{Introduction}

Breast cancer size assessment is one of the main guide for preoperative planning and is a crucial prognostic indicator (Luparia et al., 2013).Tumor size was commonly measured on digital mammography and ultrasound (US), but in the past decade breast cancer imaging has evolved considerably. In particular, magnetic resonance imaging (MRI) has been increasingly used, in addition to conventional imaging, for preoperative assessment in patients with newly diagnosed breast cancer. Furthermore in recent years MRI has emerged as the most sensitive technique for the detection and diagnosis of breast lesions, with sensitivity ranging from $94 \%$ to $100 \%$ (Orel \& Schnall 2001; Menezes, Knuttel, Stehouwer, Pijnappel \& van den Bosch 2014). Several studies have also confirmed the superior performance of MRI in tumor extent evaluation compared with digital mammography and US and its greater potential for preoperative staging, altering surgical decision in approximately $25 \%$ of the cases (Pediconi et al., 2012). In part, this can be ascribed to the density of the breast parenchyma and to the fact that mammography and US are limited in patients with dense parenchyma. However, MRI is costlier and has lower specificity as compared to full field digital mammography (FFDM), ranging from 40 to $80 \%$, moreover following EUSOMA (European Society of Breast Cancer Specialists) guidelines we are recommended to perform MRI in preoperative staging just for selected indications (infiltrative lobular carcinoma (ILC); partial breast irradiation (PBI), discrepancy between FFDM and US $>1 \mathrm{~cm}$, women at increased risk). The recent introduction of digital breast tomosynthesis (DBT) into clinical practice significantly improved the evaluation of dense breasts. DBT overcomes limitations of digital mammography, reducing both false negative and false positive rates due to tissue superimposition. (Clauser et al., 2015) Many studies conducted have shown that DBT can improve visualization of breast lesions, apart from microcalcifications(Spangler et al., 2011). DBT can also aid in determining the mammographic extent of breast cancer. Studies have shown that DBT has higher sensitivity and specificity (90\% and 79\%) when compared to FFDM (89\% and 72\% respectively) (Svahn et al., 2012; Rafferty et al., 2013). According to European guidelines, preoperative tumor size is commonly measured with FFDM and/or US and only in selected cases with MRI ( Dummin, Cox \& Plant, 2007; Sardanelli et al., 2008; Mann et al., 2008; Uematsu, Yuen, Kasami \& Uchida Y 2008; Wasif et al., 2009; Sardanelli et al., 2010; Gruber et al., 2013; Menezes et al., 2014). So far, there are currently few studies comparing DBT to MRI in preoperative staging (Luparia et al., 2013; Mariscotti et al., 2014, 2015; Kim et al., 2016). The purpose of our study was to prospectively evaluate the accuracy in tumor extent and size assessment of DBT and MRI in women with known breast cancer, with pathological size as the gold standard.

\section{Materials and Methods}

The study was approved by the ethics committee at our institution, objectives and methodology were clearly explained to the patients and all patients provided written informed consent. Each recruited woman could withdraw from the study at any time. From May 2014 to April 2016 we selected 77 consecutive women, aged between 38 and 83 years (mean age 54.3), in our prospective study. Study population included patients with newly diagnosed biopsy-proven breast cancer (Breast Imaging Reporting and Data System (BIRADS) 6), scheduled for preoperative MRI staging according to EUSOMA criteria or following our Multi-Disciplinary Team (MDT) indications. For MRI examination were excluded from the study five claustrophobic patients, three patients that required the use of a pacemaker and seven patients who had metallic implants. For DBT we excluded four patients younger than 35 years and five patients who carried gene mutation BRCA 1 or 2, due to the increased radiosensitivity (Ernestos et al., 2010; Borrego-Soto, Ortiz-López \& Rojas-Martínez 2015). Three patients with possible pregnancy were excluded both from MRI and DBT. The 50 definitive enrolled patients (all female, mean age 54.9) underwent clinical breast examination, MRI and DBT before surgery= Definitive surgery was performed in all patients from 24 hours to 1 month after completion of contrast-enhanced MRI examination, pathologic specimens (reference standard) were obtained from surgical biopsy, lumpectomy, quadrantectomy or mastectomy and were evaluated according to National Health Service Breast Screening Programme guidelines (NHSBSP 2016). Pathologic tumor size was measured on the gross specimen, on the basis of invasive component size, in-situ components were included if present.

\subsection{MRI}

All contrast-enhanced breast MRI examinations were performed on a 3.0 Tesla magnet (Discovery MR750, GE 
Medical Systems, Milwaukee, Wisconsin, USA) with a dedicated 8 channel breast coil, compatible with parallel imaging, and patients in prone position. Breast MRI protocol included transverse 2D T2-weighted sequences, transverse diffusion-weighted echo-planar sequence, transverse 3D dynamic T1-weighted sequences and sagittal post-contrast 3D T1-weighted sequence. Transverse 2D T2-weighted sequences (IDEAL) with DIXON three point technique were acquired (repetition time $9000 \mathrm{~ms}$; echo time $120 \mathrm{~ms}$, field of view $34 \mathrm{x} 34 \mathrm{~cm}$, matrix 320x224), slice thickness: $3 \mathrm{~mm}-5.0 \mathrm{~mm}$, acquisition time: $200 \mathrm{sec}$. Transverse diffusion-weighted echo-planar sequence comprising b-values of $0,800,1000$ were used; imaging parameters were: repetition time $5314 \mathrm{ms,}$ echo time $57.5 \mathrm{~ms}$, rectangular field of view $34 \times 34 \mathrm{~cm}$, flip angle $90^{\circ}$, matrix $96 \times 128$; section thickness: 3-0.5 $\mathrm{mm}$; also ADC map was acquired. Transverse 3D dynamic T1-weighted fat-sat sequences (VIBRANT) were performed before contrast agent administration and four times after contrast agent, every 2 minutes. Total acquisition time for 3D T1-weighted sequence was 120 seconds. Imaging parameters for T1-weighted sequences were identical for all patients: repetition time $8 \mathrm{~ms}$, echo time $4.3 \mathrm{~ms}$, rectangular field of view $38 \times 38 \mathrm{~cm}$, flip angle $15^{\circ}$, matrix 512x256, slice thickness: $1.2 \mathrm{~mm}$. Postcontrast three-dimensional T1-weighted dynamic MR images were acquired after administration of $0.1 \mathrm{mmol} / \mathrm{kg}$ body weight gadobenate dimeglumine (MultiHance; Bracco Imaging SpA, Milan, Italy) through an 18-gauge cannula positioned in an antecubital venous access with an automatic injector (Spectris; Medrad, Indianola, $\mathrm{Pa}$ ) at a rate of $2 \mathrm{~mL} / \mathrm{sec}$ and was followed by administration of $10 \mathrm{~mL}$ of saline at the same rate. Imaging of premenopausal women was performed between the 7th and 14th day of the menstrual cycle.

\section{$2.2 D B T$}

DBT imaging was acquired in bilateral mediolateral-oblique (MLO) and cranio-caudal (CC) projections for all patients on the dedicated FFDM system Siemens MAMMOMAT Inspiration (Siemens AG Healthcare, Erlangen, Germany). This unit has an a-Se detector with $85 \mu \mathrm{m}$ pitch and a selenium layer thickness of $200 \mu \mathrm{m}$. The A/F combinations was W/Rh and the tube voltage range was $26 \mathrm{kV}$ to $32 \mathrm{kV}$, as determined by the automatic exposure control device of the FFDM unit. The average dose to the glandular tissues within the breast (AGD) for a single DBT view was on average factor of 1.5 higher than those for a single 2D mammogram. Siemens' Breast Tomosynthesis acquires 25 images during a continuous scan, while the $\mathrm{X}$-ray source rotates along a predefined arc of $\pm 25^{\circ}$ relative to the $0^{\circ}$ position around the compressed breast, with a $2^{\circ}$ angle increment per image. Images were reconstructed using filtered back-projection, in order to provide sections parallel to the breast support, and were viewed as $1 \mathrm{~mm}$ (reconstructed) sections. DBT reconstructions were reviewed in cine or manual scroll modes on the dedicated workstation on two Nio 5-mega-pixel MDNG-6121 BARCO monitors (NYSE Euronext Brussels: BAR).

\subsection{Image Evaluation}

MRI and DBT images evaluation were performed prospectively in consensus by two breast imaging specialists (F.P., F.G.) with 15 and 7 years of experience respectively. The readers were aware of the study purpose, but they were blinded to the histological lesion location and size, considered the gold standard. MRI evaluations were performed directly at the system console using the automated software available, at this time DBT views were not available to the clinical radiologists. DBT images were interpreted at the dedicated workstation in randomly order after a 2 weeks period to avoid affecting clinical decision making. Assessment of MRI images was based on lesion morphologic characteristics and post-contrast enhancement patterns (mass or non-mass enhancement, according to ACR BI-RADS (American College of Radiology 2013)).

Assessment of DBT images was based on lesion morphologic characteristic (shape, margin, and density), calcification distribution and morphology, presence of architectural distortion or asymmetries. Breast density a,b,c,d (ACR 2013) was evaluated visually. Each lesion visible on DBT and MRI was measured using dedicated software on the workstation. The measurement was uni-dimensional, taken in the craniocaudal plane on the slice where the lesion appeared largest. MRI and DBT mass lesion size was assessed measuring tumor's core, keeping out the desmoplastic reaction surrounding the lesion. MRI non-mass lesions were measured assessing enhancement maximum extent. Architectural distortion and microcalcifications on DBT were measured acquiring lesion maximum diameter.

\subsection{Statistical Analysis}

Advanced data analyses were performed with the software R (R: A Language and Environment for Statistical Computing, R Foundation for Statistical Computing, Vienna, Austria, 2016, URL https://www.Rproject.org/). MRI and DBT sensitivity, PPV and accuracy were calculated, using histology as the gold standard. McNemar test was used to compare MRI and DBT sensitivity. Accuracy of MRI and DBT assessment was evaluated via the R package DTComPair Correlation and regression analyses was used to evaluate MRI and DBT measurements 
agreement and their dependence with respect to the histological results. Separate regression analyses were used to investigate the effect of mass or non-mass enhancement. Finally, a logistic model was fitted to the positive cases to evaluate DBT detection rate with respect to breast density, lesion size and other covariates.

\section{Results}

Of the 50 patients, in 37 patients the tumor was unifocal, in 5 multifocal, in 8 multicentric (Figure 1), for a total of 70 overall breast lesions. The 70 malignant breast lesions detected at histologic evaluation were classified according to NHSBSP 2016 guidelines as invasive ductal carcinoma $(\mathrm{n}=38)$, invasive ductal carcinoma with in situ foci $(n=12)$, ductal carcinoma in situ $(n=12)$, invasive lobular carcinoma $(n=3)$ (Figure 2), lobular carcinoma in situ with invasive component $(n=1)$, mucinous carcinoma $(n=1)$, mucinous carcinoma in situ ( $n$ $=1)$, papillary carcinoma $(n=1)$, papillary carcinoma in situ $(n=1)$. Mean, median and s.d. for the lesion size were respectively $18.8 \mathrm{~mm}, 15 \mathrm{~mm}$ and $13.6 \mathrm{~mm}$.

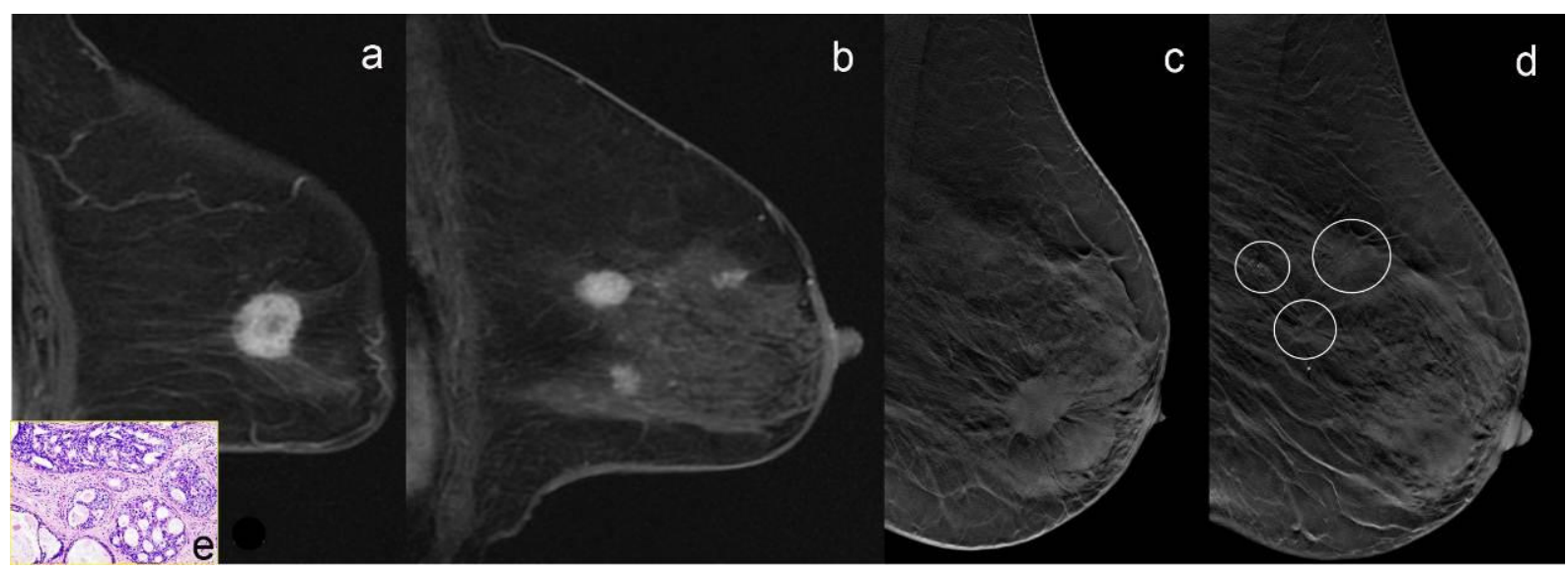

Figure 1. 50 y-o woman with multicentric disease (IDC)

T1 fat-saturated contrast-enhanced sagittal breast MRI clearly identifies four lesions on left breast (a,b), corresponding to four spiculated masses (c, d (circles)) on MLO DBT projections. MRI and DBT obtained good correlation in terms of lesions number and tumor size. Histologic evaluation showed multiple foci of invasive ductal carcinoma (e).

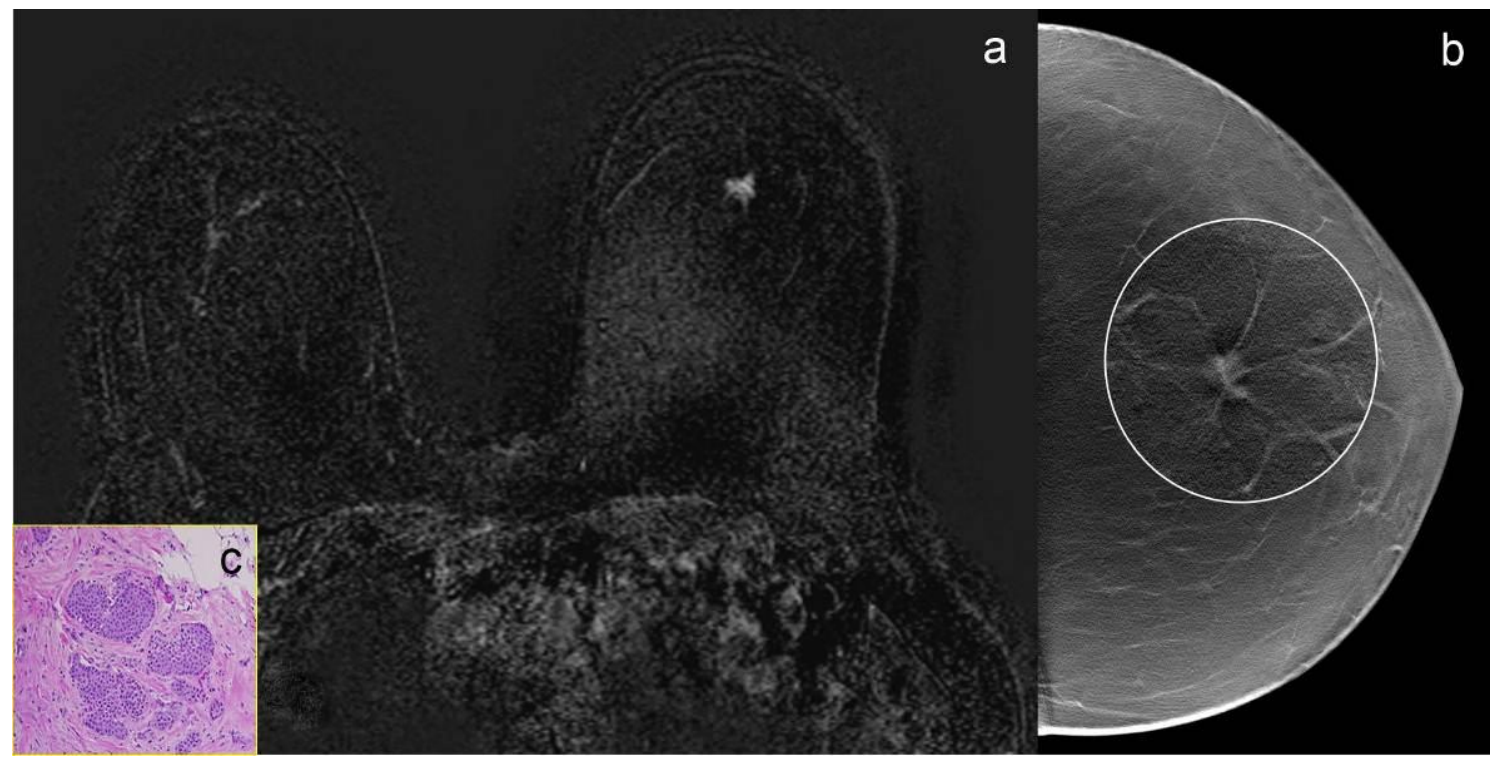

Figure 2. 65 y-o woman with unifocal invasive lobular carcinoma (ILC)

Both T1 fat-saturated contrast-enhanced axial breast MRI (a) and CC DBT (b (circle)) show a single, spiculated, $7 \mathrm{~mm}$ lesion on left breast, with high size and morphological correspondence between the two techniques. Histologic evaluation revealed an invasive lobular carcinoma (c). 


\subsection{MRI}

MRI detected 73 lesions, three of them were not confirmed at histological examination, therefore considered false positive findings. MRI had the highest level of lesion size agreement with pathology, with 57 concordant cases (81.4\%), 6 underestimations (8.6\%) and 7 overestimations (10\%). Agreement with pathology was $87.2 \%$ for $\mathrm{T} 1$ lesions, and $69.6 \%$ for $\mathrm{T} 2$ or larger lesions.

Sensitivity was equal to 1 (70/70). The positive predictive value (PPV) was equal to 0.96 , while the accuracy was 0.96 . Lesion size mean and standard deviation were $18.8 \mathrm{~mm}$ and $14.1 \mathrm{~mm}$ respectively. Pearson correlation coefficient for the lesion size (MRI versus gold standard), calculated only with the true positive cases, was 0.97 (p-value $<0.0001)$. The regression coefficient for the model where the MRI lesion size is the dependent variable and the true measurement is the covariate was equal to 1 (R-squared $=0.96$, Figure 3 (a)). Dividing the positive cases in two groups accordingly to the mass or non-mass enhancement type did not produce a significant difference in the correlation coefficients for lesion size (MRI versus gold standard, Figure 3 (d)). Anyway, fitting separate regression models to the two sub-samples lead to a significant smaller error variability for the mass enhancement sample, suggesting a more accurate evaluation of the lesion size under this group (Figure 4).

(a)

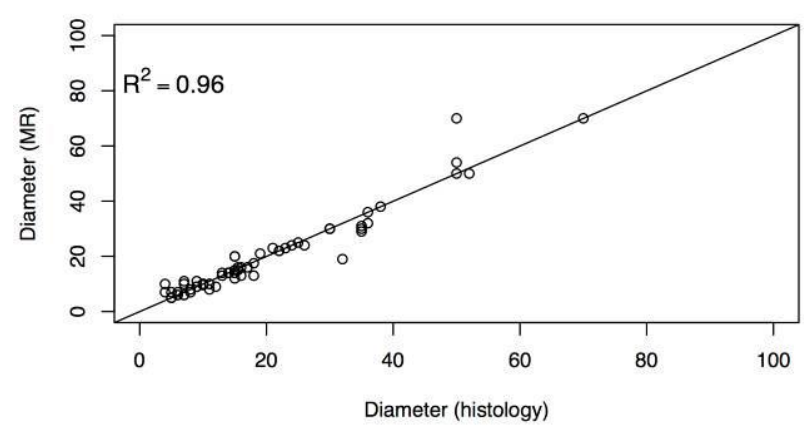

(c)

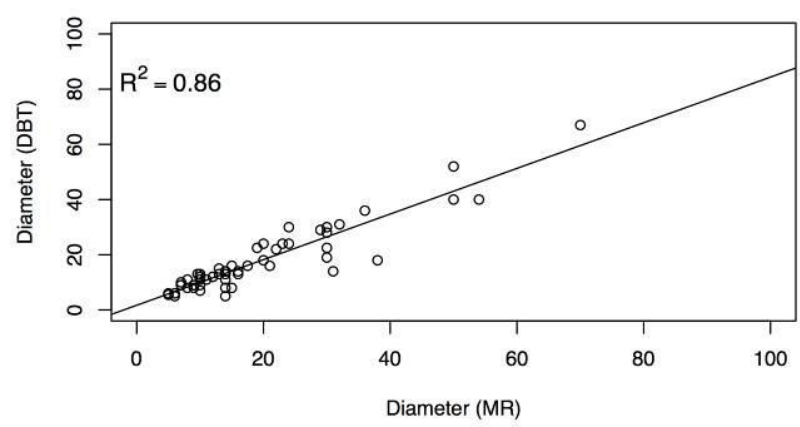

(b)

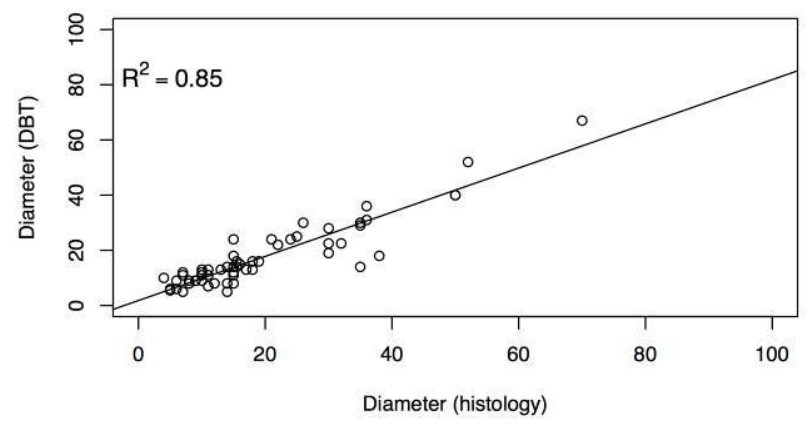

(d)

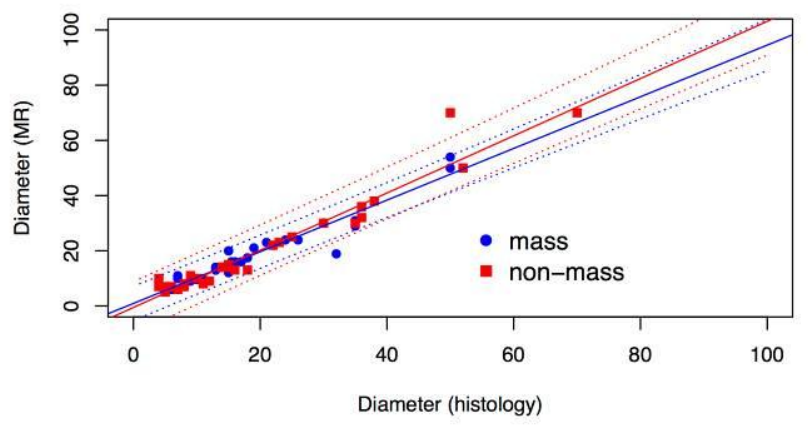

Figure 3. Lesions size assessment

Regression analysis for the diameter size, comparing MRI vs histology (a), DBT vs histology (b) and DBT vs MRI (c). Figure (d) shows the regression analysis performed dividing the lesions into the mass and non-mass groups, comparing MRI vs histology. 

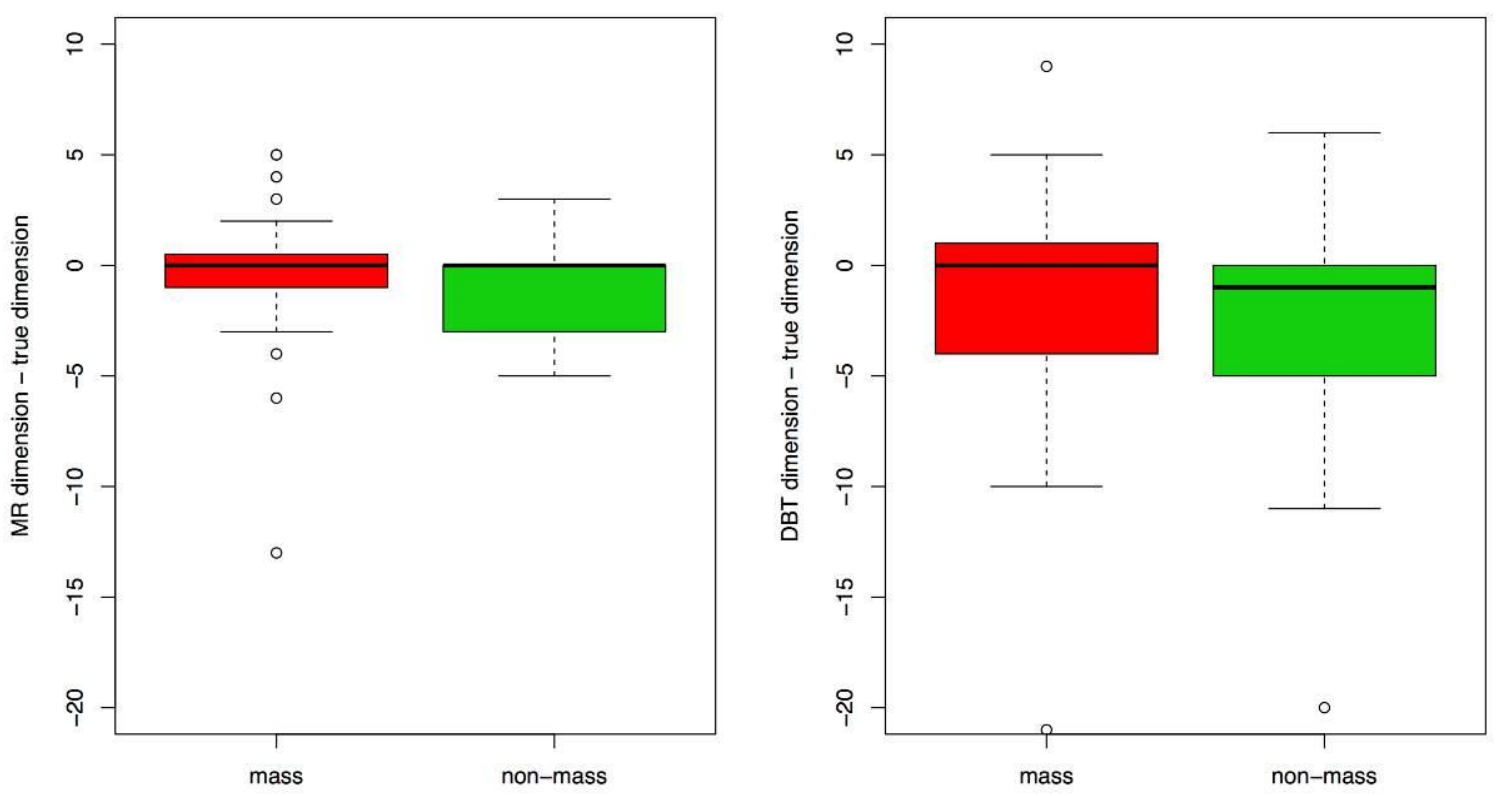

Figure 4. Error variability for mass and non-mass lesions

Box-plots representing the distribution of the difference between the estimated and the true lesion dimension, divided into the mass and non-mass groups. The box-plots have been obtained considering only the true positive cases both with MRI and with DBT. Note that in the non-mass lesions MRI underestimates the true dimension size and leads to a significantly larger measurement error respect to the mass lesions (F-test for variance comparison, p-value $=0.035$. Instead, DBT measurement error is not significantly affected by the mass or non-mass lesions type.

\section{$3.2 D B T$}

DBT identified 62 lesions, 5 of them were not confirmed by histology while 57 were true positive lesions. DBT had 33 cases $(58 \%)$ concordant in size with pathology, 18 cases were underestimated $(31.6 \%)$ and 5 were overestimated (10.5\%), while 13 lesions were not visible (18.6\%). On the basis of the histological measurements, agreement was $64.8 \%$ for lesions $\leq 2 \mathrm{~cm}$ and $45 \%$ for T2 or larger lesions. Sensitivity was equal to 0.81 (57/70), PPV was equal to 0.92 while the accuracy was 0.77 . Lesion size mean and standard deviation were $17.7 \mathrm{~mm}$ and $12 \mathrm{~mm}$ respectively. Pearson correlation coefficient for the lesion size (DBT versus gold standard), calculated only with the true positive cases was 0.92 ( $\mathrm{p}$-value $<0.0001$ ). The regression coefficient for the model where the DBT lesion size is the dependent variable and the true measurement is the covariate was equal to 0.8 (R-squared $=0.85$, Figure 3 (b)). Note that, in this case, separate regression models fitted to the mass or non-mass groups showed a not significant reduction in the prediction variability for the mass group (Figure 4).

The fitting of a logistic regression model on the positive cases assuming the DBT diagnosis as dependent variable and the true lesion size and the classification obtained accordingly on the values of the variable "density" as covariates is illustrated in Figure 5 (a). Note that the true positive rate increased with the lesion size, and higher values of the breast density provided lower detection rates. Anyway, both the covariates were not significant with the data at hand. Figure 5 (b) shows the results of a regression model with the relative error between the true lesion size and the DBT measurement is the response variable and the true size and the density value are the covariates. Observe that the relative error decreased when the true lesion size became larger and increased following the density scale. 
(a)

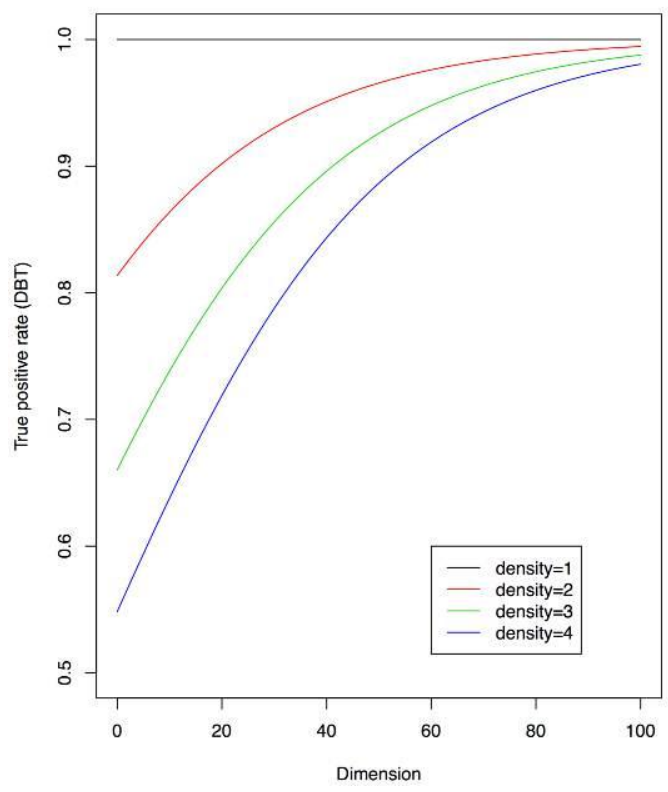

(b)

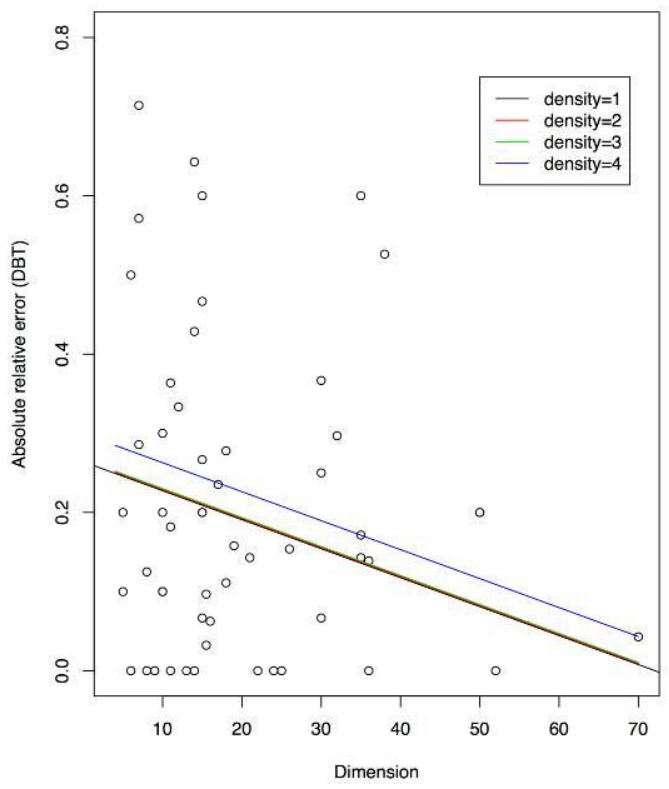

Figure 5. DBT detection rate with respect to breast density

(a) Logistic regression assuming the diagnosis with the DBT as the dependent variable and the true lesion size and "density" as the covariates. (b) Regression model assuming the diagnosis with the DBT as dependent variable and the true lesion size and "density" as covariates.

\subsection{DBT Versus MRI}

Difference in sensitivity between MRI and DBT was statistically significant (McNemar test p-value <0.001). General agreement between MRI and DBT was statistically significant (McNemar test p-value $<0.001)$. The regression coefficient for the model where the DBT lesion size is the dependent variable and the MRI measurement is the independent variable is 0.83 (R-squared $=0.86$, Figure $3(\mathrm{c})$ ).

\section{Discussion}

Breast conservative surgery, including local excision or quadrantectomy plus radiation therapy, is generally accepted as the preferable alternative to mastectomy for tumors up to $3 \mathrm{~cm}$ in size (Jatoi \& Proschan 2005). Breast cancer size represents an essential feature in order to plan the surgical treatment, therefore preoperative staging should be as accurate as possible.

In recent years, several studies promoted the role of breast MRI in addition to conventional imaging (digital mammography and ultrasound) for tumor size assessment and preoperative staging, demonstrating the great added value of this technique. However, according to EUSOMA recommendations (Sardanelli et al., 2010), only certain patients subgroups have a potential beneficial effect from preoperative breast MRI staging, therefore it should be used, in association to conventional imaging, only in selected cases. Although several studies have demonstrated the accuracy of breast MRI tumor size assessment, there are only few studies that investigate the role of DBT in preoperative staging and even less studies performing a direct comparison between DBT and MRI (Luparia et al., 2013; Mariscotti et al., 2014, 2015; Kim et al., 2016). DBT is a fast technique, with lower financial costs compared to MRI and without contraindications due to contrast agent administration or to magnetic field exposure. Moreover, evidences from many studies highlight the accuracy of DBT in the detection and evaluation of breast lesions. Our study focuses on the potential role of DBT in preoperative staging, in comparison with MRI, using final histology as gold standard. In our study, MRI showed a very high sensitivity (100\%), identifying all the malignant lesions, high PPV (96\%) and high accuracy (96\%). DBT also reported a good sensitivity (81\%), even if it was statistically significant lower than MRI (McNemar test p-value <0.001), and it also showed a good overall diagnostic performance, with good PPV (92\%) and accuracy (77\%). Our results are consistent with prior studies in literature (Luparia et al., 2013; Mariscotti et al., 2014, 2015; Kim et al., 2016). In relation to lesions measurement, MRI presented the highest agreement with pathology with a Pearson correlation coefficient of 0.97 ( $\mathrm{p}$-value $<0.0001$ ). DBT also proved to have a strong agreement with pathology, 
with a Pearson coefficient of 0.92 (p-value $<0.0001$ ), but DBT showed a tendency to underestimate larger lesions. Luparia et al., (Luparia et al., 2013) in 2013 evaluated the accuracy of tumour size assessment in preoperative staging of digital mammography, tomosynthesis, ultrasound and MRI, of 149 breast cancers in 110 patients, obtaining Pearson correlation coefficients comparable to ours ( 0.92 for MRI and 0.89 for DBT). Mariscotti et al.,(Mariscotti et al., 2014) in 2014 performed a study on 200 women with breast cancer, comparing DBT and MRI added to digital mammography and ultrasound: DBT+FFDM+US yielded a $97.7 \%$ of sensitivity, while we analyzed DBT alone reporting a lower but good value (81\%); despite high sensitivity of MRI alone (98.8\%, similar to our result), the addition of MRI to combined DBT+FFDM+US did not significantly improve sensitivity. Also considering measurement's agreement, MRI showed the strongest agreement with histology for both T1 and T2 or larger tumors (the agreement was $87.2 \%$ for $\leq 2 \mathrm{~cm}$ lesions, and $69.6 \%$ for $>2 \mathrm{~cm}$ lesions, as already reported by Luparia et al (Luparia et al., 2013) and Onesti et al., ( Onesti, Mangus, Helmer \& Osland 2008)). DBT, instead, confirmed the worse measurement in larger lesions, with agreement of $64.8 \%$ for lesions $\leq 2 \mathrm{~cm}$ and $45 \%$ for T2 or larger lesions, these results are substantially consistent with literature (Luparia et al., 2013), even with slightly lower percentages due to our small number of patients.

No significant difference in mass and non-mass lesion's measurement correlation coefficients was identified, however the mass group showed a smaller error variability suggesting a more accurate measurement of lesions with a mass morphology respect to non-mass group. This result was statistically significant only for MRI, while for DBT the error variability was similar between the two groups, even if higher in non-mass group. This result is probably due to the worse performance that DBT reported also in mass lesions measurement, therefore the difference in the evaluation of the two groups was smaller. In our study we analyzed also the impact of breast density in the evaluation of lesion detection rate and size on DBT. We found a lower detection rate and an higher relative error in the evaluation of lesion size for patients with dense breasts (ACR c, d), in particular for small lesions, as already reported in literature (Mariscotti et al., 2014). In case of large lesions DBT performed similarly in both dense and fatty breasts (ACR a, b).

Our study presented some limitations that are the small number of patients and the selected population, because we enrolled only women with diagnosed breast cancer.

\section{Conclusion}

In conclusion, MRI confirmed to be the most accurate imaging technique in preoperative staging of breast cancer. However, DBT showed very good accuracy, sensitivity and tumor size assessment and could be a valid tool for preoperative staging when MRI should be avoided because of patients' clinical conditions or any contraindications to MRI. Moreover, considering the fewer contraindications and the higher availability of DBT, further studies could investigate the potential role of contrast-enhanced digital breast tomosynthesis in pre-operative staging in order to approach MRI in terms of breast lesion morphologic and vascular characterization.

\section{Acknowledgements}

The authors state that this work has not received any funding.

\section{References}

American College of Radiology (2013). ACR BI-RADS® Atlas 5th Edition

Borrego-Soto, G., Ortiz-López, R., \& Rojas-Martínez, A. (2015). Ionizing radiation-induced DNA injury and damage detection in patients with breast cancer. Genet Mol Biol., 38, 420-432. https://doi.org/10.1590/S1415-475738420150019

Clauser, P., Carbonaro, L., Pancot, M., Girometti, R., Bazzocchi, M., Zuiani, C., \& Sardanelli, F. (2015). Additional findings at preoperative breast MRI: the value of second-look digital breast tomosynthesis. Eur Radiol., 25, 2830-2839. https://doi.org/10.1007/s00330-015-3720-5

Dummin, L. J., Cox, M., \& Plant, L. (2007). Prediction of breast tumor size by mammography and sonography--A breast screen experience. Breast, 16, 38-46. https://doi.org/10.1016/j.breast.2006.04.003

Ernestos, B., Nikolaos, P., Koulis, G., Eleni, R., Konstantinos, B., Alexandra, G., ... Hoeijmakers, J. (2010). Increased chromosomal radiosensitivity in women carrying BRCA1/BRCA2 mutations assessed with the G2 assay. Int J Radiat Oncol Biol Phys., 76, 1199-205. https://doi.org/10.1016/j.ijrobp.2009.10.020

Gruber, I., Rueckert, M., Kagan, K., Staebler, A., Siegmann, K., Hartkopf, A., ... Hahn, M. (2013). Measurement of tumour size with mammography, sonography and magnetic resonance imaging as compared to histological tumour size in primary breast cancer. BMC Cancer, 13, 328. 
https://doi.org/10.1186/1471-2407-13-328

Jatoi, I., \& Proschan, M. (2005). Randomized trials of breast-conserving therapy versus mastectomy for primary breast cancer: a pooled analysis of updated results. Am J Clin Oncol., 28, 289-94. https://doi.org/10.1097/01.coc.0000156922.58631.d7

Kim, W., Chang, J., Moon, H., Yi, A., Koo, H., Gweon, H., \& Moon, W. (2016). Comparison of the diagnostic performance of digital breast tomosynthesis and magnetic resonance imaging added to digital mammography in women with known breast cancers. Eur Radiol., 26, 1556-1564. https://doi.org/10.1007/s00330-015-3998-3

Luparia, A., Mariscotti, G., Durando, M., Ciatto, S., Bosco, D., Campanino, P., ... Gandini, G. (2013). Accuracy of tumour size assessment in the preoperative staging of breast cancer: comparison of digital mammography, tomosynthesis, ultrasound and MRI. Radiol Med., 118, 1119-1136. https://doi.org/10.1007/s11547-013-0941-z

Mann, R., Veltman, J., Barentsz, J., Wobbes, T., Blickman, J., \& Boetes, C. (2008). The value of MRI compared to mammography in the assessment of tumour extent in invasive lobular carcinoma of the breast. Eur J Surg Oncol., 34, 135-42. https://doi.org/10.1016/j.ejso.2007.04.020

Mariscotti, G., Houssami, N., Durando, M., Campanino, P., Regini, E., Fornari, A., ... Gandini, G. (2015). Digital Breast Tomosynthesis (DBT) to Characterize MRI-Detected Additional Lesions Unidentified at Targeted Ultrasound in Newly Diagnosed Breast Cancer Patients. Eur Radiol., 25, 2673-2681. https://doi.org/10.1007/s00330-015-3669-4

Mariscotti, G., Houssami, N., Durando, M., Bergamasco, L., Campanino, P., Ruggieri, C., ... Gandini, G. (2014). Accuracy of Mammography, Digital Breast Tomosynthesis, Ultrasound and MR Imaging in Preoperative Assessment of Breast Cancer. Anticancer Res., 34, 1219-1226

Menezes, G., Knuttel, F., Stehouwer, B., Pijnappel, R., \& van den Bosch, M. (2014). Magnetic resonance imaging in breast cancer: A literature review and future perspectives. World J Clin Oncol., 5, 61-70. https://doi.org/10.5306/wjco.v5.i2.61

Onesti, J. K., Mangus, B. E., Helmer, S. D., \& Osland, J. S. (2008). Breast cancer tumor size: correlation between magnetic resonance imaging and pathology measurements. Am J Surg., 196, 844-850. https://doi.org/10.1016/j.amjsurg.2008.07.028

Orel, S. G., \& Schnall, M. D. (2001). MR Imaging of the Breast for the Detection, Diagnosis, and Staging of Breast Cancer. Radiology, 220, 13-30. https://doi.org/10.1148/radiology.220.1.r01j13113

Pediconi, F., Miglio, E., Telesca, M., Luciani, M., Kirchin, M., Passariello, R., \& Catalano, C. (2012). Effect of Preoperative Breast Magnetic Resonance Imaging on Surgical Decision Making and Cancer Recurrence Rates. Investigative Radiology, 47(2), 128-135. https://doi.org/10.1097/RLI.0b013e318230061c

Rafferty, E., Park, J., Philpotts, L., Poplack, S., Sumkin, J., Halpern, E., \& Niklason, L. (2013) Digital Mammography and Breast Tomosynthesis Compared with Digital Mammography Alone: Results of a Multicenter, multireader trial. Radiology, 266, 104-113. https://doi.org/10.1148/radiol.12120674

Sardanelli, F., Giuseppetti, G. M., Canavese, G., Cataliotti, L., Corcione, S., \& Cossu, E. (2008). Indications for breast magnetic resonance imaging. Consensus Document "Attualità in Senologia, Florence 2007. Indicazioni alla risonanza magnetica mammaria. Documento di Consenso "Attualità in senologia\&quot; In: La Radiologia Medica. Springer, 1085-1095

Sardanelli, F., Boetes, C., Borisch, B., Decker, T., Federico, M., Gilbert, F., ... Wilson, R. (2010). Magnetic resonance imaging of the breast: Recommendations from the EUSOMA working group. Eur J Cancer, 46, 1296-1316. https://doi.org/10.1016/j.ejca.2010.02.015

Spangler, M., Zuley, M., Sumkin, J., Abrams, G., Ganott, M., Hakim, C., ... Gur, D. (2011). Detection and Classification of Calcifications on Digital Breast Tomosynthesis and 2D Digital Mammography: A Comparison. Am J Roentgenol, 196, 320-324. https://doi.org/10.2214/AJR.10.4656

Svahn, T., Chakraborty, D., Ikeda, D., Zackrisson, S., Do, Y., Mattsson, S., \& Andersson, I. (2012) Breast tomosynthesis and digital mammography: A comparison of diagnostic accuracy. $\mathrm{Br} \mathrm{J}$ Radiol., 85, https://doi.org/10.1259/bjr/53282892

Uematsu, T., Yuen, S., Kasami, M., \& Uchida, Y. (2008). Comparison of magnetic resonance imaging, multidetector row computed tomography, ultrasonography, and mammography for tumor extension of 
breast cancer. Breast Cancer Res Treat, 112, 461-474. https://doi.org/10.1007/s10549-008-9890-y

Wasif, N., Garreau, J., Terando, A., Kirsch, D., Mund, D., \& Giuliano, A. (2009). MRI versus ultrasonography and mammography for preoperative assessment of breast cancer. Am Surg., 75, 970-5

\section{Copyrights}

Copyright for this article is retained by the author(s), with first publication rights granted to the journal.

This is an open-access article distributed under the terms and conditions of the Creative Commons Attribution license (http://creativecommons.org/licenses/by/4.0/). 